Volume 10, No.1, January - February 2021

International Journal of Advanced Trends in Computer Science and Engineering

Available Online at http://www.warse.org/IJATCSE/static/pdf/file/ijatcse501012021.pdf

https://doi.org/10.30534/ijatcse/2021/501012021

\title{
A Predictive Meta Model for Forecasting Stock Price using Time Series Data
}

\author{
Md. Iftekharul Alam Efat ${ }^{*}$, Rahat Uddin Azad, KhairAhammed \\ Institute of Information Technology Noakhali Science and Technology University Noakhali, Bangladesh \\ Correspondent Email: iftekhar.efat@gmail.com
}

\begin{abstract}
Stock market prediction through time series is a challenging as well as an interesting research area for the finance domain, through which stock traders and investors can find the right time to buy/sell stocks. However, various algorithms have been developed based on the statistical approach to forecast the time series for stock data, but due to the volatile nature and different price ranges of the stock price one particular algorithm is not enough to visualize the prediction. This study aims to propose a model that will choose the preeminent algorithm for that particular company's stock that can forecast the time series with minimal error. This model can assist a trader/investor with or without expertise in the stock market to achieve profitable investments. We have used the Stock data from Stock Exchange Bangladesh, which covers 300+ companies to train and test our system. We have classified those companies based on the stock price range and then applied our model to identify which algorithm suites most for a particular range of stock price. Comparative forecasting results of all algorithms in diverse price ranges have been presented to show the usefulness of this Predictive Meta Model.
\end{abstract}

Key words: Stock Market Prediction, Forecasting, Time Series, Decision System, Trend Estimation.

\section{INTRODUCTION}

A stock or share market refers to the interchange marketplace where buying, selling and publicly shareable companies share issuance takes place. It has a great impact on the financial market economy and investors want to make some profit from that. These also include securities embedded on a stock exchange so that the investors could choose one or several companies' shares to buy and gain more profit effortlessly.

Basically, stock prices are defined by their market share and transaction volume [1]. If a share is traded more means the share has more important to the traders and it will be more valuable. Contrary wise, the less traded share value decreases for having less importance to the traders [2]. Therefore, the important fact is if the share market direction can be predicted successfully, it will be easier for the investors to make trading decisions. Moreover, predicting the future values of the share market is an essential task while making the correct decision whether to buy or sell the share.

Nevertheless, it is one of the most difficult tasks to predict stock market trends. Because there are several economic factors like economic outlook, interest rate, economic policy changes, and inflation that can affect the market trends [1]. These factors lead to the stock market being nonlinear and dynamic. In the stock market, prediction techniques can play a large role in gathering investors. For decades, investors depend on some heuristic strategy for stock trend prediction and ensure risk-free trading. But the dynamic and nonlinear nature makes it complex and harder to predict through some heuristic strategy [2]. As a result, it leads the investors in taking wrong decisions for buying or selling stocks, and later that can lead them to enormous loss.

Eventually, many researchers are trying to develop a trustworthy and intelligent decision-making system, which will predict the nonlinear stock trends for the investors. They proposed some statistical approaches like Experience Sampling Method (ESM), Auto-Regressive Integrated Moving Average (ARIMA), regression and variants of regression, machine learning, sentiment analysis, and some hybrid model to predict the stock trends [3-6]. But still, it is not suitable for real-time data or live testing and there are multiple factors behind this like price variation, noisiness, and fake news. Therefore, in a simulated environment, it is still intangible.

Moreover, an interesting thing is these approaches by the researchers have self-defeating nature means that if an approach can predict good then sharing it will render the novel approach useless. In recent years, sentiment analysis gets a lot of attention because of the social media influences and researchers have focused more on Twitter analysis [5]. But social media is not reliable enough and there may have fake or biased news, which can mislead the prediction result. Most of the studies focused on a single algorithm and consider a small portion of the stock market for the prediction, which can mislead the prediction model.

However, the real-time stock prediction is a major challenge. Also, because of the nonlinear nature, the estimation of the trend might be impossible in some aspects [7]. Moreover, researchers are more focused on short-term prediction rather than long-term prediction. Alongside the nonlinear nature of these data makes it quite a complicated task to plot these stocks' trendiness time series and predict 
real-time stock prices. Although according to numerous reports stock prices are not just randomly generated values. The behavior of stock prices can be dealt with discrete-time series depends on a set of well-defined numerical data points [8]. Thus, many data mining and statistical approaches are playing important role in the stock market to find hidden patterns and increasing a certain level of forecasting accuracy. But the challenges are still immutable.

Again, the advancement of the statistical approach is very much efficient allowing time series data to extract important information and forecast the trendiness. ARIMA is used to analyze and predict future stock prices based on the patterns of historical time series data. ARIMA can handle the non-stationary time series data and convert it to stationary data before making a prediction [8]. Moreover, ARIMA gives more authentic results than most of the other algorithms for direct forecasting. Again, considering the repeating nature and seasonal effect [9], exponential smoothing [10] is a family of forecasting methods, which has been applied for smoothing time series data. Moreover, triple exponential smoothing is known as Holt Winter's Exponential Smoothing which creates a trend line for the data and seasonal indices to forecast more accurately [11].

From previous studies, it can be assumed that stock price forecasts are based on data available to the public that have some predictive relationship with future stock returns [12]. After exploring various stock values from various stock exchange websites; where there are some common date-wise values like (opening, closing, highest, lowest, average), etc. Furthermore, these huge amounts of data are generated by various websites forced the researchers to apply data mining techniques to make investment decisions. Since it is very much important to find a solution to this challenging problem analyzing the stock market trendiness with proper and enough information for making the decision safer.

Therefore, we focused on the close-range values and predict thereby. We approached multiple time series algorithms (ARIMA, SES, SARIMAX, etc.) by the range values and noticed that relying on a single algorithm can't be a great decision. We have found that multiple algorithms perform best with respect to the close-range values and we proposed a Meta model that will train multiple time series algorithms, make a comparison based on the error rate, and finally, the model with the least error rate will be selected. We considered the listed companies in Dhaka Stock Exchange (DSE) [13].

This predictive Meta model is able to identify the best prediction model algorithm of various ranges with minimal error. Considering multiple algorithms allows the model to be robust on stock prediction and provides more accurate results for pricing decisions.

The structure of the article is as follows: Section 2 highlights literature reviews on the stock price prediction. Section 3 puts forward the model to forecast stock trends with minimum error in detail. Section 4 reports the data set and narrates the results of the experiment. At the very end, the conclusion and future scope are described in Section 5.

\section{LITERATURE REVIEW}

Stock price fluctuation of a company is the main concern of the investors while buying/selling stock and this is one of the most emerging issues in the business research arena. "Efficient Market Hypothesis (EMH)" and "Random Walk Theory" specify that stock price maintains a random walk process and it's impossible to predict [14]. But researchers argue that EMH fails to explain some of the weird stock market characteristics. They claimed that especially the existing developing country's stock market is not maintaining independence. Therefore, investors can use the information that is not yet incorporated by the market for making better financial plans.

Additionally, statistical forecasting is based on the implementation of historical data to find the pattern and predict future trends. Dutta in 2012 classified companies based on the previous year's performance into 'good' and 'bad' categories [15]. The author applied the logistic regression model on the financial ratios (net sales, P/B (price-to-book), book value, etc.), and gained $74.6 \%$ accuracy. The paper contributed to understanding the forecasting of the condition of companies.

In 2013, Devi proposed an approach of the ARIMA model over four of the Indian midcap companies [16]. There, they showed that the Nifty 50 index should be the choice of new investors because of its low volatility and error rate. In 2014, Ariyo used the ARIMA model for stock forecasting of Nokia and Zenith Bank [17]. Identifying the best models among all the generated model R-square, standard error of the regression, Bayesian information methodology are chosen by the author.

Again, Bhuriya et al predicted the stock prices of Tata Consultancy Services [18]. They considered five predictor features (open, low, high, close price, and volume) and the author implemented several regression models (linear, polynomial, and radial basis function-RBF) and compare the performance metric values based on the confidence level of each model. Bhuriya showed that linear regression outperformed among them with a confidence level of $97 \%$ [18]. Also, In 2019, Idrees analyzed and forecast the Indian stock exchange using the ARIMA model [3]. Their approach performed brilliantly and the mean percentage error was roughly 5\%. They performed "ADF" and "L-jung box" tests for validating their model.

However, machine learning has been used widely for its capability and potentiality in stock market prediction. Especially, deep learning and neural network-based approaches are becoming more and more popular for their strong capability of finding patterns. Consequently, Dey et al in 2016 adopted the eXtreme Gradient Boosting (XGBoost) algorithm on stocks data of apple and yahoo for long-term forecasting [19]. Similarly, Singh et al predict the trends of google stock prices considering some technical indicators as the feature their model achieves $87-99 \%$ accuracy using the deep neural network (DNN) [4]. 
Later, in 2017, Avaram employed the convolutional neural network (CNN) algorithm to predict the Finnish stock exchange and achieved $67.38 \%$ precision [20]. The same year, Samarawickrama [21] employed Recurrent Neural Network (RNN), and Selvin [22] employed CNN, RNN, LSTM to Sri-Lankan stock data, and Infosys stock data respectively. They proved that deep learning approaches are very much capable of identifying inter-relationships in the trends. In 2018 Zhang proposed a random forest model for predicts the volatility of the stock markets [23]. This model experimented on historical data of SGEM (Shenzhen Growth Enterprise Market) in China and historical stocks data classifies into four classes (up, down, flat, and unknown) before train the model. Nevertheless, in 2019, Varun [24] used the combination of Relative Strength Index (RSI) and Long Short-Term Memory (LSTM) and achieved 92\% accuracy. Correspondingly, deep learning approaches are also adopted for better forecasting by Kim and $\mathrm{Yu}$, where they found that the candlestick chart performs best in stock prediction with deep models $[25,26]$.

Recently, sentiment analysis also has major impacts on stock prediction. The idea of sentiment analysis is based on the impacts of share markets according to the news or reactions of the people. It can lead to fluctuations in a short time-period therefore predicting in an earlier stage could help the investors making business plans. In 2011, Bollen conducted a twitter analysis for predicting the Doe Jones Industrial Average (DJIA) closing prices [5]. They employed a Self-Organizing Fuzzy Neural Network (SOFNN) and achieved $87.6 \%$ accuracy.

Consecutively, in 2014, Akira predicts the stocks of Nikkei companies by analyzing newspaper articles [27]. In 2015, Nardo predicts stock market trends summarizing online news literature [28]. Also, Cakra and Trisedya proposed an approach over the Indonesian stocks data that can predict stock prices, price fluctuations based on sentiment analysis [29]. They classified the data into three categories: 'positive', 'neutral', and 'negative' and achieved $67.37 \%$ accuracy on predicting the price fluctuation.

Again, Pagolu in 2016 employed Word2vec (2-layer NN) and $\mathrm{N}$-gram to analyze the sentiments of tweets and applied the random forest classifier for price prediction [30]. They achieved $70 \%$ accuracy also reported that the correlation between sentiments and price is $71.82 \%$. In 2019, Maqsood et al. found that volatility of stock markets has nonlinear nature, it depends upon the economic and political conditions of the country as well as on community sentiments [31]. But also, not all the major events have an impact on prediction. They proposed a system that does sentiment analysis and forecast stock trends.

However, many of the researchers also proposed a hybrid model for forecasting the stock market trends. In 2016, Chen proposed a hybrid model, the combination of two pattern recognition techniques (PIP and template matching) for finding patterns on the National Association of Securities Dealers Automated Quotations (NASDAQ) indices and Taiwan Capitalization Weighted Stock Index (TAIEX) [32]. Likewise, Wei proposed an inference system based on the Empirical Mode Decomposition (EMD) hybrid model called Adaptive Network-based Fuzzy inference system (ANFIS) for trends identification of the Taiwan stock exchange [6].

Consequently, Liu [7] was identical in closing price prediction with a hybrid model including empirical wavelet transform (EWT) based decomposition, LSTM neural network, and outlier robust extreme learning machine (ORELM) [7]. Later, in 2020, Dai used 3 of the well-known technical indicators with a two-step economic constraint model to generate buy/sell signals based on mathematical functions [33]. An overview of state-of-art techniques for stock market prediction is displayed in Table 1.

In summary, a trading rule, based on flag pattern recognition, which is focused on both the opening and closing data of the Dow Jones Industrial Average (DJIA) is the most challenging in time series data [34]. However, stock price follows the random walk theory but it can also be repeated and stock price is biased by price and volume. Hence, price (open, high, low, and close) and stock transactions volume time series data are sufficient for forecasting the trends and the periodic projection of the stock price can be helpful in predictions.

Table 1: State-of-art Forecasting Techniques for Stock Price Prediction

\begin{tabular}{|c|c|c|c|}
\hline Authors & Year & Area/Market & Forecasting Technique \\
\hline Akira Yoshihara [27] & 2014 & $\begin{array}{l}\text { Tokyo Stock Exchange } \\
\text { (Nikkei companies) }\end{array}$ & $\begin{array}{l}\text { Recurrent Temporal Restricted Boltzmann } \\
\text { Machine (RTRBM), RNN-RBM }\end{array}$ \\
\hline Ritika Singh \& Shashi Srivastava [4] & 2016 & Google stock prices & DNN \\
\hline Liang-Ying Wei [6] & 2016 & Taiwan Stock Exchange & $\begin{array}{l}\text { Adaptive Network-Based Fuzzy Inference } \\
\text { System (ANFIS) }\end{array}$ \\
\hline AvraamTsantekidis [20] & 2017 & Finland Stock Exchange & $\mathrm{CNN}$ \\
\hline A.J.P. Samarawickrama [21] & 2017 & Sri Lankan Stock Exchange & RNN \\
\hline SreelekshmySelvin [22] & 2017 & Infosys stock data & CNN, RNN, LSTM \\
\hline Taewook Kim, Ha Young Kim [26] & 2019 & SPDR S\&P 500 ETF & LSTM and CNN \\
\hline Hui Liu, Zhihao Long [7] & 2020 & S\&P 500, CMSB, DJI & $\begin{array}{l}\text { EWT based decomposition, LSTM neural } \\
\text { network, ORELM based error correction }\end{array}$ \\
\hline Zhifeng Dai [33] & 2020 & Stock Market Analysis & $\begin{array}{l}\text { Combination of technical indicators (EMA, RSI, } \\
\text { KDJ) and two-step economic constraint }\end{array}$ \\
\hline
\end{tabular}




\section{PROPOSED METHOD}

Our proposed approach illustrated in Figure $\mathbf{1}$ is divided into two phases. In the first phase, the stock price of a particular company has been divided into train and test data, and then run through a different predictive algorithm to forecast the stock price. The various forecasted stock price has been compared with the test data to calculate the error (deviation). From those various error rates, the least error algorithm has been chosen as the best-suited algorithm and then loads the whole data to train with that particular algorithm and forecast the stock price for the next month.

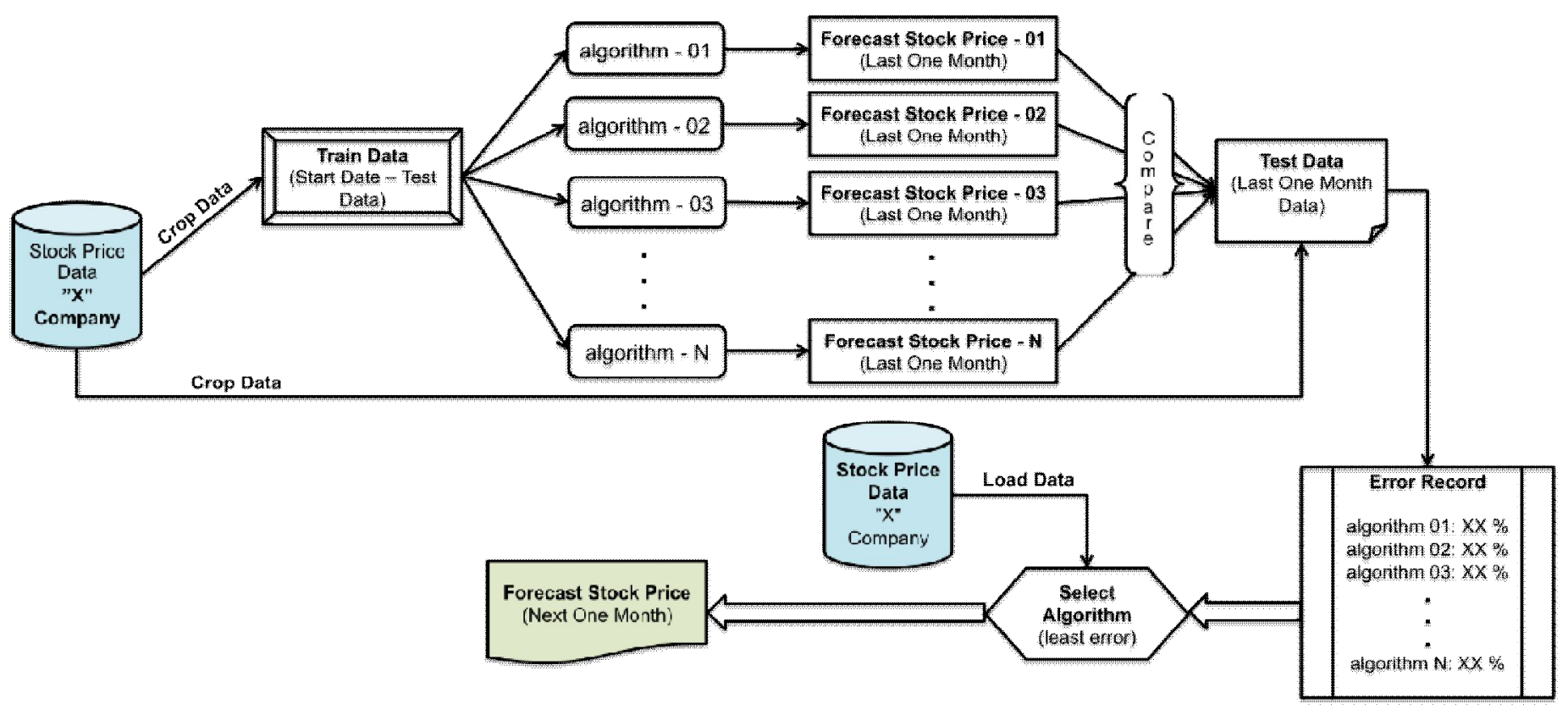

Figure 1: The Framework of the Predictive Meta Model

\subsection{Autoregressive Integrated Moving Average (ARIMA) Model}

The autoregressive integrated moving average (ARIMA) model is widely used as a time series model, which has a better understanding of the time-series data to forecast future values in the series [35]. This model claims that the time series data have to be stationary or not, then it can be made to be stationary through differencing it [36].

Generally, the notation ARIMA $(p, d, q)$ indicates to non-seasonal ARIMA models where three parameters $\mathrm{p}, \mathrm{d}$, and $\mathrm{q}$ are non-negative integers. The number of time lags of the AR model is denoted by $p$, the degree of differencing is denoted by $\mathrm{d}$ which is the number of required differencing to make the time series stationary, and $q$ is the order of the MA term. However, the forecasting equation of the ARIMA model is a linear equation in which the predictors consist of lags of the forecast errors. To forecast using ARIMA model is defined as:

$$
\begin{gathered}
y_{t}=c+€_{1} y_{t-1}+€_{2} y_{t-2}+\ldots+€_{p} y_{t-p}+\Omega_{1} \epsilon_{t-1} \\
+\Omega_{2} \epsilon_{t-2}+\ldots+\epsilon_{t}
\end{gathered}
$$

However, ARIMA doesn't require any predictor variables and can show better results than regression if fitted perfectly. Also, it can handle non-stationary time series data well but can be terrible in the random walk time series data.

\subsection{Seasonal Autoregressive Integrated Moving Average Exogenous (SARIMAX) Model}

Adapting from the ARIMA model, another robust and exponential model SARIMAX, is pretty much the same as the SARIMA model with an external predictor variable [37]. We can express the external variables $\mathrm{X}$ as the multilinear regression equation.

$$
Y_{t}=\beta_{0}+\beta_{1} X_{1},+\ldots+\beta_{k} X_{k, t}+\omega_{t}
$$

There, $X 1, t \quad, \quad X 2, t \quad, \quad \ldots X k, t$ and $B 0, \quad B 1, \ldots . \quad B k$ is the observations parameter and regression coefficients of external variables corresponding to $\mathrm{Yt}$, dependent variable. Moreover, $\omega t$ is stochastic residual, which can be expressed in ARIMA form

$$
\omega_{t}=\frac{\theta_{q}(B) \theta_{Q}\left(B^{s}\right)}{\varphi_{\mathrm{p}}(B) \varphi_{P}\left(B^{s}\right)(1-B)^{d}\left(1-B^{s}\right)^{D}} \varepsilon_{t}
$$

Where the order of differencing, the order of seasonal differencing is indicated by $\mathrm{d}$ and $\mathrm{D}$. The seasonal autoregressive order, respectively $\mathrm{P}$ and $\mathrm{p}$. Similarly, the seasonal moving average denotes the non-seasonal autoregressive order, the non-seasonal moving average is denoted by respectively $\mathrm{Q}$ and $\mathrm{q}$. Moreover, $\mathrm{s}$ is the seasonal length and B is the lag operator. Finally, the equation will be: 


$$
\begin{aligned}
Y_{t}=\beta_{0}+\beta_{1} X_{1, t} & +\ldots+\beta_{k} X_{k, t} \\
& +\frac{\theta_{q}(B) \theta_{Q}\left(B^{s}\right)}{\varphi_{\mathrm{p}}(B) \varphi_{P}\left(B^{s}\right)(1-B)^{d}\left(1-B^{s}\right)^{D}} \varepsilon_{t}
\end{aligned}
$$

The main advantage of the SARIMAX model is the capability of handling both stationary and non-stationary time-series data. That means it can handle the seasonality of the trends. Also, the SARIMAX model has the predictor variables that can find useful information or significant occurrence from the outliers.

\subsection{Holt Winter's Exponential Smoothing (HWES) Model}

Holt Winter's Exponential Smoothing (HWES) is commonly known as triple exponential smoothing which basically implement by applying exponential smoothing three times. First of all, this model calculates a trend line for the data then for weighted the values in that trend line it calculates seasonal indices based on where the time frame falls within a certain cycle of a certain length [38].The forecasting model HWES can be defined as:

$$
\begin{aligned}
& \text { level } L_{t}=\alpha\left(y_{t}-S_{t-s}\right)+(1-\alpha)\left(L_{t-1}+b_{t-1}\right) \\
& \text { trend } b_{t}=\beta\left(L_{t}-L_{t-1}\right)+(1-\beta) b_{t-1} \\
& \text { seasonal } S_{t}=\gamma\left(y_{t}-L_{t}\right)+(1-\gamma) S_{t-s} \\
& \text { forecast } F_{t+k}=L_{t}+k b_{t}+S_{t+k-s}
\end{aligned}
$$

There seasonal cycle length of the model is denoted by $s$ for interval $0 \leq \alpha \leq 1,0 \leq \beta \leq 1$ and $0 \leq \gamma \leq 1$. At that time in the seasonal cycle the difference between the current level and the data is calculated by seasonal equation. Then later the same point in the cycle the calculated difference is added to a forecasting equation.

However, at least one complete seasonal cycle $y_{1}, y_{2}, \ldots . y_{s}$ is needed to initialize the seasonality. Moreover, without observing one complete cycle forecasts cannot be made. Therefore, $L_{s}, b_{s}$ and $S_{1}, S_{2}, \ldots S_{s}$ is used in the above formulae to make forecast from time $s$ onwards. In fact, sometimes it's best to complete two cycles with initialize the trend for make better forecast. Sensible starting values are:

$$
\begin{gathered}
L_{s}=\frac{1}{S} \sum_{i=1}^{S} y_{i} \\
b_{s}=\frac{1}{S}\left[\frac{y_{s+1}-y_{1}}{S}+\frac{y_{s+2}-y_{2}}{S}+\cdots+\frac{y_{2 s}-y_{s}}{S}\right] \\
S_{i}=y_{i}-L_{S}, \quad i=1, \ldots S
\end{gathered}
$$

HWES model allows a user smoothing the time series data and forecasting thereby. Exponential smoothing assigns exponentially decreasing weights to the time series data that means older data will have less weight than the most recent data. The only problem is the smoothing technique will lag behind the actual trendiness.

\subsection{Simple Exponential Smoothing (SES) Model}

Exponential smoothing is a time series forecasting technique using the exponential window. Exponential smoothing technique works upon past prediction errors [39]. The equation of simple exponential smoothing is defined as:

$$
y_{t}=y_{t-1}+\alpha\left(x_{t}-y_{t-1}\right)
$$

Where $\alpha$ the ratio of smoothing factor and the value of $\alpha$ should be between 0 to 1 . The smoothed statistic is defined as $y_{t}$ and the previous smoothed statistic is defined as $y_{t-1}$. On the other hand, $y_{t}$ is a simple weighted average of the current observation $x_{t}$

Moreover, the term smoothing coefficient $\alpha$ is type of indicator which actually affect the level of smoothing like larger values of $\alpha$ actually reduce the level of smoothing, and in other case output series is just the current observation when $\alpha=1$. Besides when two observations are available then this model can easily apply and it produces a smoothed statistic.

Essentially, to produce the best fit the value of $\alpha$ is chosen by minimizing the sum of the squared in-sample forecast errors. A research study of Dimson and Marsh shows that if the optimal value of $\alpha$ was chosen in every month then it will provide better forecasting result [10].

\subsection{Vector Autoregressive Model:}

The Vector Auto regression (VAR) model is an extension of the univariate autoregressive model and most successful model for the multivariate time series analysis. The structure of VAR models is flexible and it is often useful for describing the dynamic behavior of economic and financial time series data[40]. Moreover, in the VAR model it is possible to make conditional on specified variables in the model, which provide better forecasting result to those from univariate time series models.

The notation VAR indicates vector autoregressive model of order one. By considering a two variable VAR with one lag The VAR (1) model is defined as,

$$
\begin{aligned}
& y_{1, t}=c_{1}+\phi_{11,1} y_{1, t-1}+\phi_{12,1} y_{2, t-1}+e_{1, t} \\
& y_{2, t}=c_{2}+\phi_{21,1} y_{1, t-1}+\phi_{22,1} y_{2, t-1}+e_{2, t}
\end{aligned}
$$

Where, noise processes $e_{1, t}$ and $e_{2, t}$ is may be correlated in a contemporary way. On the other hand influence of the $\ell$ th lag of variable $y_{i}$ on itself and $y_{j}$ on $y_{i}$ captured by the coefficient $\phi$ ii, $\ell$ and $\phi_{\mathrm{Ij}, \ell}$.

In addition, sometimes the forecast variable of VAR model is influenced by the predictor variables and imposes a unidirectional relationship that is considering a limitation of that model.

\subsection{Prophet Forecasting Model:}

Prophet Forecasting Model is another popular and widely used time series forecasting model that addresses the weekly or yearly cycle, holidays, outliers and trend change of the data and then predict the future points (forecasts) [9].The main advantage of this model is that it is capable of adjust the seasonality with multiple time steps alongside allows the analyst to make different assumptions about seasonal trends. Also, there is no need to estimate assumptions are regularly spaced, and neither needs to interpolate missing values.

This model is employed with a simple modular regression model that is often fitted well with the default parameters as well as adjustment of those parameters based on type or data for forecasting. Another component is responsible for improving the forecast accuracy that flag the forecast for 
rechecking the part and making improvements gradually.

By explicitly defining change-points $\left(\gamma_{j}\right)$ their model combines trend changes in the growth model where rates of the growth are allowed to change. However, their trend model is actually based on a saturating growth function that can be defined as:

$$
g(t)=\frac{C(t)}{1+\exp \left(-\left(k+a(t)^{T} \delta\right)\left(t-\left(m+a(t)^{T} \gamma\right)\right)\right)}
$$

Where $C(t)$ is the time-varying capacity, k the growth rate, and $\mathrm{m}$ an offset parameter.

The presented logistic growth model is single type of sigmoid curve and a special type of generalized logistic growth curves. Prophet is robust to missing data, seasonality in the trends and large outliers and also it will be fitted well on historical data and thus will give better accuracy.

\subsection{Trend Estimating Linear Regression (TELR) Model}

A decomposable time series model based on prophet forecasting model, using linear regression has been proposed. This model has three significant components trend, seasonality and holidays [41], can be described in the following equation:

$$
y(d)=g(d)+s(d)+h(d)+\alpha(d)
$$

Where, the functions $g(d), s(d), h(d)$ represents respectively trend, seasonality and holiday effects of the TELR model.

Trend function that models non-periodic changes in time series values and $s(d)$ function capture periodic changes (e.g., weekly and yearly seasonality). On the other hand, $h(d)$ function calculates the effects of holidays which occur on potentially irregular schedules over one or more days. Then any types of idiosyncratic changes which are not assume by the model are captured by the error term $\alpha(d)$.

However, to prepare the growth function, a piecewise linear regression has been used that could be defined as:

$$
g(d)=\left(l+\frac{\alpha(d)}{\alpha}\right) d+\left(q+\frac{\alpha(d)}{\gamma}\right)
$$

Where, $\alpha$ is the rate adjustments, 1 is the growth rate, $n$ is the offset, and $\gamma_{j}$ is set to $-s_{j} \alpha_{j}$ for treating as a continuous function. The change points $s_{j}$ could be selected randomly from a candidate list. We use the prior $\alpha_{j} \sim \operatorname{Laplace}(0, \Omega)$ of the changepoints. $\Omega$ maintains the model flexibility.

Piecewise Linear Regression (Algorithm 1) starts with on boarding data and store them into a data frame and then 'trend', a dictionary key added. Then comes the piecewise linear function evaluation part, $g(d)$ with $\mathrm{d}$ (date), $\alpha$ (changing rate at each point), $p, q, s_{j}$ (change time). $\gamma$, the intercept changes. Finally, the cumulative slope and intercept $(\mu)$ has been achieved with respect to date (d). In that manner, a time series array $A, \forall k=1 \ldots . t$ has been defined that returns array of one.

Later on, the intervals and seasonality have been created from data-frame. $\hat{y}$ prediction intervals and stock trend return data-frame with uncertain intervals on the forecasting techniques. Here, train_y is the class label.

$$
\text { train_y }=\text { seasonal }+ \text { trend }
$$

At last, $y(d)$, the predicted value with respect to datehas been calculated. Finally, the linear trend model gives the stock price-forecasting outcome.

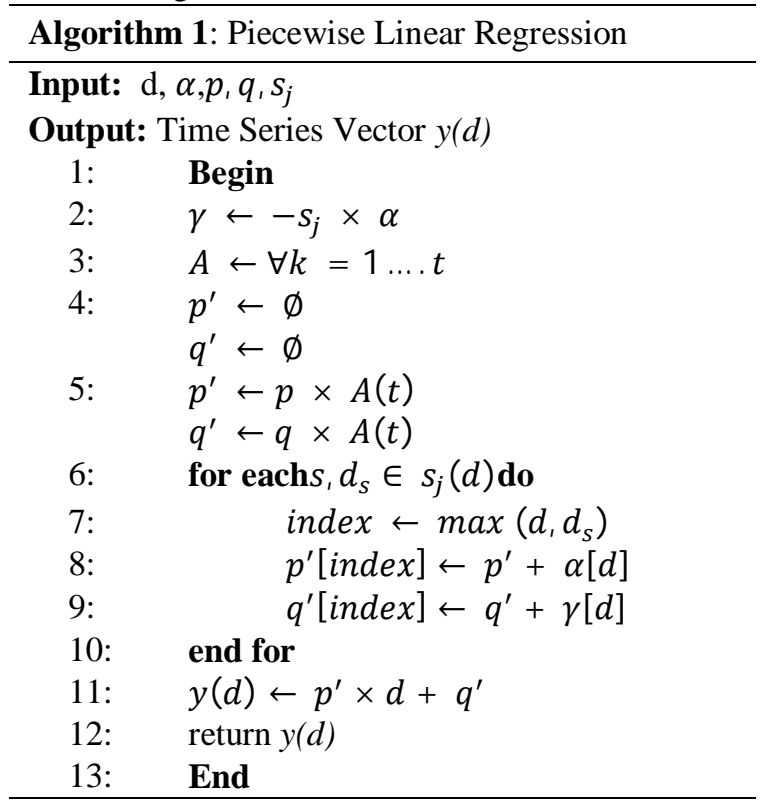

This model comes up with very much robust and accurate in prediction considering multiple trend parameter for indicating the peaks and linearity of the time series data.

\subsection{Predictive Meta Model}

This predictive Meta model is designed for forecasting stock data, which could be fitted with any kind of time series model or state-of-art forecasting algorithm, pictorial view is in Figure 1.

This model starts with loading the stock data: from the starting date to till date, of a company. Then the data is cropped into two part: one part is last 30 days data for test and the rest part is kept for train the state-of-art algorithms. The train and test data are denoted by $\zeta^{R}$ and $\zeta^{T}$ respectively, in Algorithm 2.

Next, the train data is fitted as $M_{i}$, with each time series algorithm to tune that respective algorithm with fixing parameters and noise values, which is expressed as $\varepsilon_{i}$.

After that, each individual algorithm will forecast $\left(\mathrm{F}_{i}\right)$ the next 30 days stock price (starting after the last date of train data). Now, each forecasting price will be compared with the test data, and a forecasting performance measurement matrix, $\delta_{A}$ has been calculated that actually find the error rate, which is later used to choose the best fitted algorithm for that particular company's stock data.

Now, based on the error rate, the least error algorithm has been selected to forecast stock price of that company. Then, again the data is loaded to train the algorithm, but this time, the total previous data: from start date to till date has been given as input. Finally, a forecast, $\mathrm{F} \leftarrow\left\{\left\{D_{n+1}, P_{1}\right\},\left\{D_{n+2}, P_{2}\right\}, \ldots \ldots\left\{D_{n+30}, P_{30}\right\}\right.$ will 
be produced with predicting the stock price of that company with minimal error.

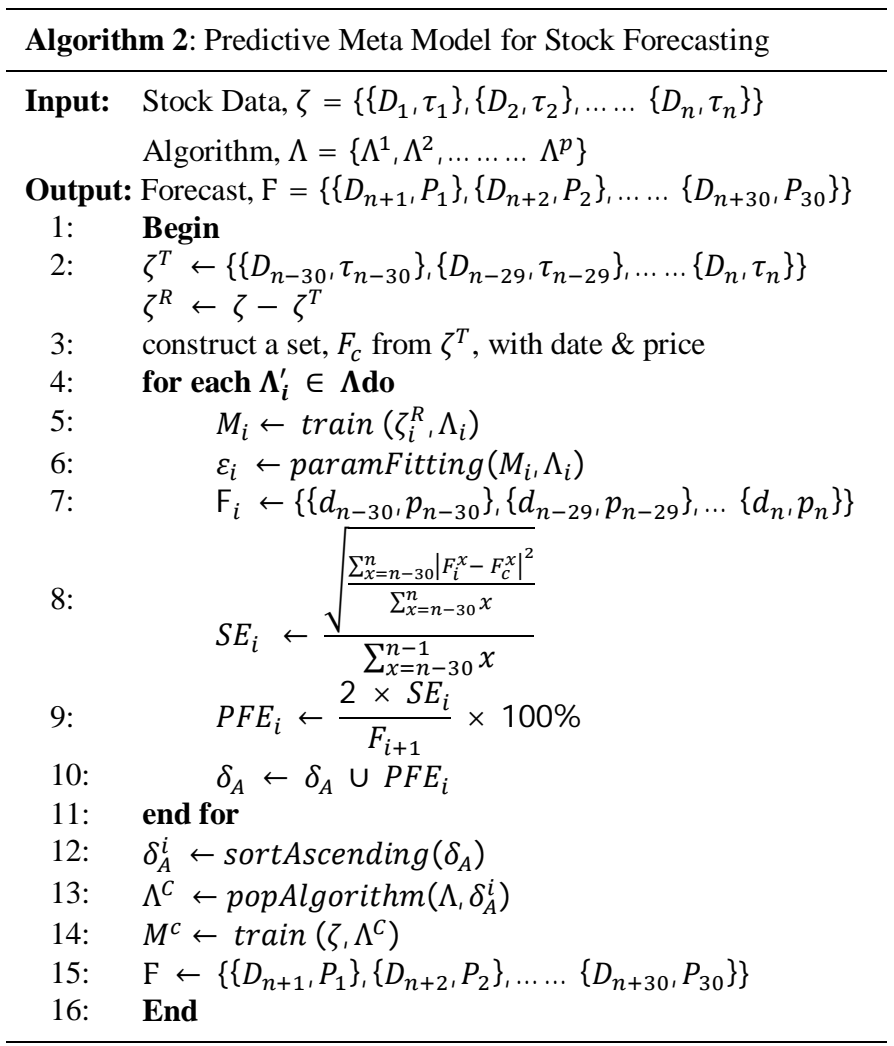

\section{EXPERIMENTAL RESULT \& DISCUSSION}

In the course of the experiment, the proposed Meta model has experimented with the open-access data from Stock Bangladesh [13]. This experimental design was employed to evaluate the proposed Meta model.

\subsection{Experimental Setup}

To experiment with the overall model the following materials are required.

Workstation: At the time of the experiment the workstation configuration was:

- Operating System: Windows 10 Enterprise

- Processor: Intel(R) Core (TM) i5-7200U CPU @ $2.50 \mathrm{GHz}$

- Memory: 8 GB DDR3

- Secondary Storage: SSD 224GB (Flash Storage)

- Graphics: Intel(R) HD Graphics 6208073 MB

Python: Python is the fastest-growing well-known programming language for built-in support for scientific computing as well as in the field of research and development works. Python has a wide variety of libraries like NumPy, SciPy, Pandas, and Matplotlib, which are extremely optimized, well documented, and easy to compute. Most importantly it has strong support for data preprocessing and to better handle the data deluge.
Jupyter Notebook: To work with python, we used Jupyter Notebook, which is an open-source web application. It is extremely useful for data cleaning, processing, transformation, normalization, numerical simulation, visualization, and statistical modeling. Alongside it allows us to write expressive code, present our findings and embed results/graphs in the same document as with code.

\subsection{Dataset}

We collected the stock price data from an open-source website Stock Bangladesh [13]. This website contains the historical stock data of all the companies listed on the Dhaka Stock Exchange (DSE). This historical data includes different financial sectors like Insurance, Ceramics, Cement, Engineering, Tannery, Textile, Telecommunications, Travel, etc.

Further, we have collected much popular Bangladeshi company's stock price data like:

Prime Bank, Saif Power, South East, BBS Cables, Nploymar, Aramit Cement, Linde Bangladesh Limited, Grameenphone, Dutch Bangla Bank Limited (DBBL), Rahim Textile Company, IbnSina, etc. from 1st December 2018 to 25 December 2020. Due to the covid-19 pandemic in the Bangladesh stock market the year 2019-2020 had been very challenging.

After collecting the dataset, we have considered the close value as an indicator for divide the whole dataset into multiple ranges single dataset. Then from every single dataset, we have extracted 10 companies' data and convert it into an individual single dataset. Later for each company, data from December 2018 to October 2020 used as the training dataset, and November 2020 was used as the test dataset.

A sample used data of BBS Cables Company has been displayed in Table 2. It represents several important attributes of the stock market along with companies' information and their stock prices. The details of those attributes are given below.

Open: In the financial markets the term "Open" has several usages. Basically, the opening price is the price at the beginning of trading sessions or other words first trade value. Moreover, for the day's trading activity the opening price is a significant indicator as well as for those who are looking for short-term results like daily traders.

Close: The term "Close" refers to the ending price of a stock in a financial market or other words the last price of trading sessions. This term helps investors to analyze historical returns on investment and understand the changes in the value over a time frame.

High: The term "High" means the highest price in a given period of time. Broadly high intraday refers to high business value during the whole trading session. Sometimes it may or may not be higher than the "Open" or "Close" value.

Low: The term "Low" means the lowest price in a given time-period. It is typically lower than the "Open" and "Close" value.

Volume: Volume is the total amounts of shares bought or sold off any stock in a given period of time. In the larger sense volume is the total number of stocks that are actually traded during the trading day. It is important because the more 
volume the more people agree with the price of the stock and also an important indicator in technical analysis.

Table 2: Sample Data (BBS Cables Stock Data)

\begin{tabular}{cccccc}
\hline Date & Open & High & Low & Close & Volume \\
\hline $10-01-19$ & 72.36 & 73.36 & 71.27 & 72.45 & 246105 \\
$10-02-19$ & 72.82 & 72.91 & 71.91 & 72.18 & 182444 \\
$10-03-19$ & 72.45 & 72.64 & 71.55 & 71.82 & 179627 \\
$10-06-19$ & 72 & 72 & 71.09 & 71.36 & 155922 \\
$10-07-19$ & 71.64 & 71.64 & 69.45 & 69.64 & 256857 \\
$10-09-19$ & 69.73 & 69.73 & 65.36 & 65.91 & 490989 \\
$10-10-19$ & 67.09 & 67.09 & 63.55 & 63.91 & 427414 \\
$13-10-19$ & 64.64 & 65.64 & 63.36 & 63.82 & 297580 \\
$14-10-19$ & 64 & 65.18 & 63.27 & 64.36 & 292522 \\
$15-10-19$ & 64.82 & 70.82 & 64.82 & 70.82 & 806610 \\
$16-10-19$ & 71.55 & 72.64 & 67.64 & 68.55 & 767654 \\
$17-10-19$ & 68.55 & 68.55 & 66.18 & 66.55 & 373765 \\
$20-10-19$ & 66.64 & 68.09 & 66.55 & 67 & 276455 \\
\hline
\end{tabular}

We considered the close column as the label or target column and train the system for stock trendiness estimation. Further, some auxiliary columns are considered for improving the model prediction accuracy.

\subsection{Forecasting Performance Evaluation}

It is mandatory for every forecasting model to evaluate its forecast accuracy without which it is difficult to determine how good a model performs on unstable and new data set. Further, the residual's size is not a dependable indication because it can't depict the size of forecast errors. So, forecasting accuracy can only be estimated by the size of errors rather than the size of residuals. For evaluating the error size there have many performance measurement techniques.

Root Mean Square Error (RMSE):We know that the average of the squares of the errors is measured by using the Mean Square Error (MSE). The MSE is calculated as the average of the squares sum value of the errors [42]. Therefore, the standard deviation of the residuals (prediction errors) is called Root Mean Square Error (RMSE). Moreover, RMSE is a measure of how residuals spread out as well as these residuals are a measure of how far from the data points of the regression line.

$$
R M S E=\sqrt{\frac{\sum\left(Y_{t}-F_{t}\right)^{2}}{N}}
$$

Where time-period is denoted by $t$, the number of periods predicted refers by $\mathrm{N}$, and in the time-period $\mathrm{t}$ actual value is denoted by Yt. Ft for predicted value in time-period t. RMSE is a process of measurement to check how wide out these residuals are. In other words, it gives an account of how to centralize the data is around the line of best fit.

Mean Absolute Percentage Error (MAPE):Recently Mean Absolute Percentage Error is a very popular technique for evaluating forecasting methods. Which is actually a statistical evaluation technique of a forecast system and computes error as a percentage. Basically, for each time period, the average absolute percentage error minus actual values and divided those values by actual values is equal value to this method [42]. The MAPE is calculated as:

$$
M A P E=\frac{\sum \frac{\left|Y_{t}-F_{t}\right|}{Y_{t}}}{n} \times 100 \%
$$

Where actual value is denoted by $\mathrm{Yt}$ and $\mathrm{Ft}$ is the predicted value. Being unit-free is one of the best advantages of MAPE, therefore it is frequently used to compare forecasting performances of different forecasting algorithms. Based on the MAPE measurement a scale was proposed by Lewis [43] to judge the model where High Accuracy is considered for less than 10\% MAPE value, between $11 \%$ and $20 \%$ (inclusive) is a good forecast, between $21 \%$ to $50 \%$ (inclusive) is reasonable forecasting and more than $50 \%$ MAPE value represents inaccurate forecasting.

Percentage Forecast Error (PFE):The traditional forecasting performance measurement techniques doesn't have any real-world business meaning or context that encourages developing new measurement technique called Percentage Forecast Error (PFE). It is a new forecasting alternative that offers a practical and accurate measurement as well as forecaster's confidence. The PFE is calculated as,

$$
P F E=\frac{2 \times S_{d}}{\hat{Y}_{t+1}} \times 100 \%
$$

There, standard error is denoted by $\mathrm{S}_{\mathrm{d}}$, and in the time period $t+1$ the forecasted value is denoted by $\hat{Y} t+1$. Moreover, this PFE measurement technique is quite similar to the coefficient of variation $(\mathrm{CV})$. Because the coefficient of variation $(\mathrm{CV})$ measures the ratio of the standard deviation to the mean. Basically, the standard error multiplied by 2 that's why the difference is in the numerator of the PFE. Therefore, the measurement output is 2 times deviate from the standard. Using the PFE value with a high level of certainty, which is almost $95 \%$ and it, allows one to say that next time period forecast will be within the actual value PFE\%[44].

\subsection{Result Analysis\& Discussions}

It has been observed that the performance of a time series model varies from different stock price ranges, therefore, we have classified stock companies into different sub-group. However, analyzing the stock market in Bangladesh, it has been observed that price range can be grouped as like: 0-50 taka, 50-100 taka, 100-200 taka, 200-300 taka, 300-400 taka, and $400+$ taka.

We have investigated the stock company price based on the above groups and found the result interestingly. The lower, the stock price is the performance is riskier and for high price stock, the prediction-actual difference is not much significant statistically.

For the experiment, we have presented Prime Bank as a lower bound group of 0-50-taka range. Similarly, Meghna Cement Company Limited, Atlas Bangladesh Company Limited, Rahim Textile Company Limited, Grameen Phone, and Linde Company Limited as 50-100, 100-200, 200-300, 
$300-400$, and up to 400 taka range respectively. These companies' cases are taken considering the seasonal effect, vibrant change, financial challenges, and uncertainty issues; therefore, our proposed Meta model is justified to compete for these challenges.

Our Meta model actually decides the time series model based on the minimal error, therefore, sample forecasting comparing with the actual value has been exhibited in Figure 2. There are 6 different graphs from different stock price ranges, as explained earlier. Also, a comparison table has been displayed in Table 3 with the error rate for a different model (algorithm) in the variant price range. In this graph, the actual values of the stock are marked as blue with a bold line as well as, in each sub-graph the selected algorithm is also showed in bold line to understand how close that model (algorithm) can forecast the data comparing with the actual price. However, in the first three sub-graph, Figure 2 (part: a, b, c) the TELR method forecasts better as the price range of the stock is small in range.

Again, for the sub-graph in Figure 2 (part: d) we have found that HWES forecasts much better, but if we look at the error rate from Table 3, we will found the minimum PFE for the stock price range (200-300) is $1.81 \%$ and this PFE is almost same for three different algorithms: ARIMA, PROPHET, and HWES. Hence, our Meta model looked for the other forecasting performance measurement metric to choose the better forecasting algorithm. Here, MAPE values are $2.93 \%, 2.87 \%$, and $4.04 \%$ for ARIMA, SES, and HWES algorithm respectively, similarly, the RMSE values are 3.91, 3.63, and 3.62

Comparing the PFE, MAPE, and RMSE values, it can be identified that, HWES algorithm suits much for forecasting that particular company's stock price Also, if we look at the sub-graph in Figure 2 (part: d), we will found that, the nature and growth of the train data (December 2018 to October 2020) is abruptly changed on November 2018, therefore the TELR or SES algorithm could not forecast with the nearest value, but if we consider the up or down growth of the line curve, we will see the similarity of upwards or downwards trend is almost same as the actual trend but due to the gap of the stock price, the PFE, MAPE, or RMSE values are higher than ARIMA, PROPHET, or HWES.

Next, is the stock price range of $300-400$, from the values of Table 3 the PFE is almost similar $(1.42 \%, 1.39 \%, 1.31 \%)$ for ARIMA, PROPHET, and TELR algorithm. Hence, our Meta model considers the MAPE and RMSE value, in this case, comparing all these performance forecast errors, the PROPHET algorithm has been selected. However, from the sub-graph in Figure 2 (part: e), both the trend line is drawn from PROPHET and TELR is almost similar to the actual line. Hence, it could be concluded that for trend line estimation PROPHET and TELR works better than other algorithms.

Similarly, for stock price range up to 400 , we have found that PROPHET forecasts much better, but if we look at the PFE error rate from Table 3, again we will find the minimum PFE is almost the same for HWES, PROPHET, and TELR algorithm. Hence comparing the PFE, MAPE, and RMSE values are proposed Meta model identified that the PROPHET algorithm is a better forecasting model for predict that particular company's stock price.

Therefore, it can be concluded that with the most unstable and challenging stock value of Bangladeshi companies' this Meta model performs preeminent to identify which algorithm will be best suited for a particular company's stock price prediction. Also, another contribution TELR method is justified considering the performance in almost all variation and challenges of the stock price.

Table 3: Comparison of Forecast Performance Measurement (error) of Different Model in Variant Stock Price Range

\begin{tabular}{lccccccccc}
\hline \multirow{2}{*}{ Algorithm } & \multicolumn{3}{c}{$\mathbf{0 - 5 0}$} & \multicolumn{3}{c}{$\mathbf{5 0 - 1 0 0}$} & \multicolumn{3}{c}{$\mathbf{1 0 0 - 2 0 0}$} \\
\cline { 2 - 10 } & $\mathbf{R M S E}$ & MAPE & PFE & RMSE & MAPE & PFE & RMSE & MAPE & PFE \\
\hline ARIMA & 0.321203452 & $4.31 \%$ & $0.73 \%$ & 1.803948569 & $5.31 \%$ & $1.18 \%$ & 4.592789052 & $4.71 \%$ & $1.02 \%$ \\
SARIMAX & 0.329112027 & $4.98 \%$ & $0.73 \%$ & 1.667107045 & $6.23 \%$ & $1.19 \%$ & 4.312265822 & $5.54 \%$ & $0.86 \%$ \\
HWES & 0.404973471 & $5.17 \%$ & $0.79 \%$ & 1.766032453 & $5.67 \%$ & $1.22 \%$ & 4.879358305 & $3.93 \%$ & $1.15 \%$ \\
SES & 0.532546119 & $9.63 \%$ & $1.09 \%$ & 1.635346561 & $6.15 \%$ & $1.19 \%$ & 7.462459742 & $6.26 \%$ & $1.08 \%$ \\
PROPHET & 0.412086343 & $6.11 \%$ & $0.85 \%$ & 1.856124674 & $5.59 \%$ & $\mathbf{0 . 9 2 \%}$ & $\mathbf{3 . 9 2 2 8 3 8 1 3 6}$ & $\mathbf{3 . 1 7 \%}$ & $\mathbf{0 . 2 5 \%}$ \\
VAR & 0.330473322 & $4.15 \%$ & $0.82 \%$ & 1.78714612 & $5.08 \%$ & $1.21 \%$ & 4.992885889 & $4.03 \%$ & $0.92 \%$ \\
TELR & $\mathbf{0 . 2 9 7 0 4 3 1 6 5}$ & $\mathbf{3 . 7 8 \%}$ & $\mathbf{0 . 6 1 \%}$ & $\mathbf{1 . 6 2 7 7 5 1 9 4 2}$ & $\mathbf{4 . 0 2 \%}$ & $\mathbf{0 . 9 1 \%}$ & $\mathbf{3 . 8 9 2 3 8 2 1 6 2}$ & $3.31 \%$ & $\mathbf{0 . 2 7 \%}$ \\
\hline & $\mathbf{2 0 0 - 3 0 0}$ & & & $\mathbf{3 0 0 - 4 0 0}$ & & & $\mathbf{4 0 0 +}$ & \\
Algorithm & $\mathbf{2}$ & $\mathbf{M A P E}$ & $\mathbf{P F E}$ & $\mathbf{R M S E}$ & $\mathbf{M A P E}$ & $\mathbf{P F E}$ & $\mathbf{R M S E}$ & $\mathbf{M A P E}$ & PFE \\
\hline ARIMA & 3.914728238 & $2.93 \%$ & $\mathbf{1 . 8 5 \%}$ & 13.66256965 & $\mathbf{3 . 6 3 \%}$ & $\mathbf{1 . 4 2 \%}$ & 20.02694128 & $3.52 \%$ & $2.25 \%$ \\
SARIMAX & 4.563458714 & $4.11 \%$ & $2.01 \%$ & 15.97973537 & $4.27 \%$ & $2.98 \%$ & 22.10627273 & $4.71 \%$ & $3.56 \%$ \\
HWES & $\mathbf{3 . 6 3 6 9 3 9 2 9 3}$ & $4.04 \%$ & $\mathbf{1 . 8 1 \%}$ & $\mathbf{1 1 . 9 2 3 8 2 5 4 9}$ & $4.96 \%$ & $1.75 \%$ & $\mathbf{1 4 . 2 2 6 4 7 2 7 9}$ & $3.79 \%$ & $\mathbf{1 . 9 7 \%}$ \\
SES & 8.618348954 & $3.71 \%$ & $2.03 \%$ & 19.13635578 & $3.76 \%$ & $4.79 \%$ & 24.68040831 & $5.81 \%$ & $2.54 \%$ \\
PROPHET & $\mathbf{3 . 6 2 8 3 7 3 2 5 5}$ & $\mathbf{2 . 8 7 \%}$ & $\mathbf{1 . 8 6 \%}$ & $\mathbf{1 1 . 8 6 4 1 8 4 9 3}$ & $\mathbf{3 . 5 7 \%}$ & $\mathbf{1 . 3 9 \%}$ & $\mathbf{1 4 . 6 3 3 7 9 9 5 1}$ & $\mathbf{2 . 4 5 \%}$ & $\mathbf{1 . 9 1 \%}$ \\
VAR & 4.520758912 & $3.49 \%$ & $2.96 \%$ & 19.12753072 & $4.67 \%$ & $3.54 \%$ & 18.59238611 & $3.44 \%$ & $2.63 \%$ \\
TELR & 4.033761926 & $3.58 \%$ & $1.97 \%$ & 12.52398232 & $5,54 \%$ & $\mathbf{1 . 3 1 \%}$ & 14.94612312 & $2.82 \%$ & $\mathbf{1 . 9 5 \%}$ \\
\hline
\end{tabular}




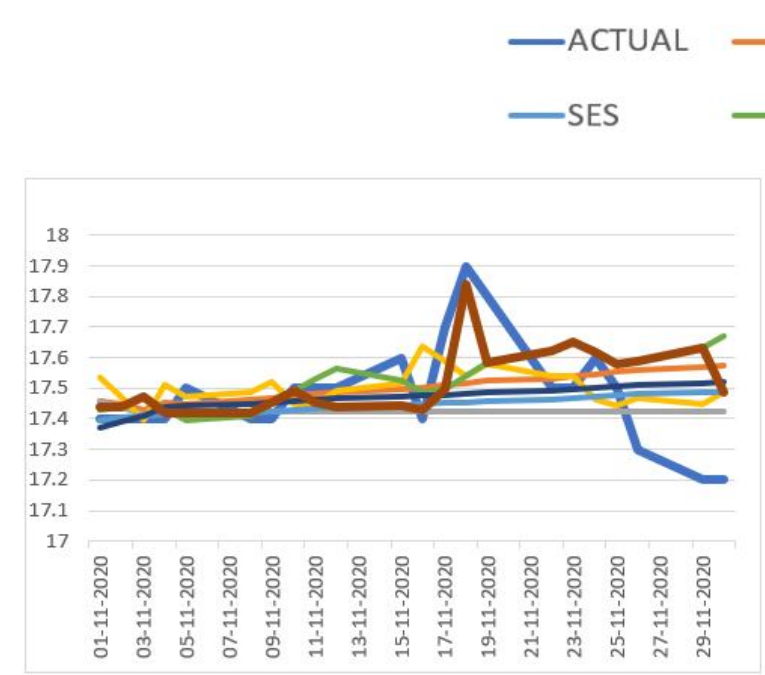

(a) 0-50 - Prime Bank Limited

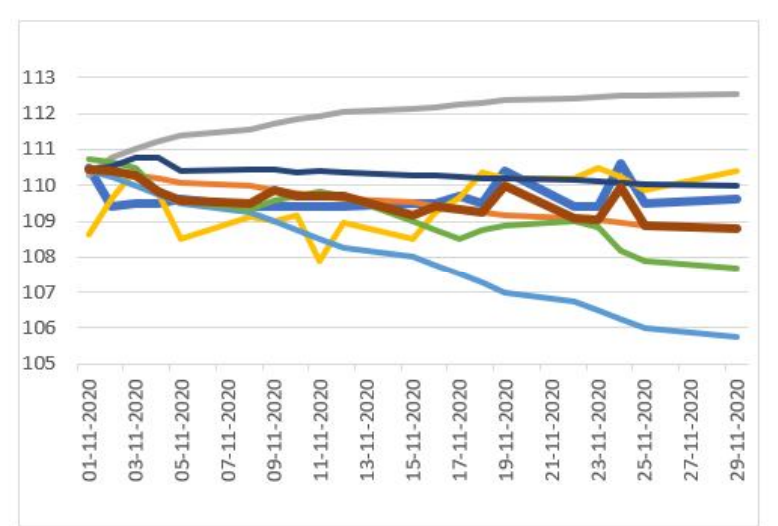

(c) 100-200 - Atlas Bangladesh Company Limited

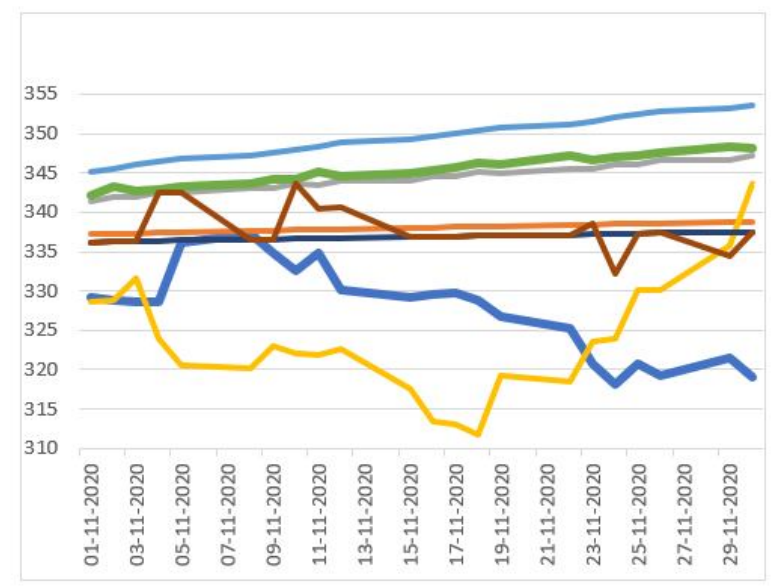

(e) 300-400 -Grameen Phone Company Limited
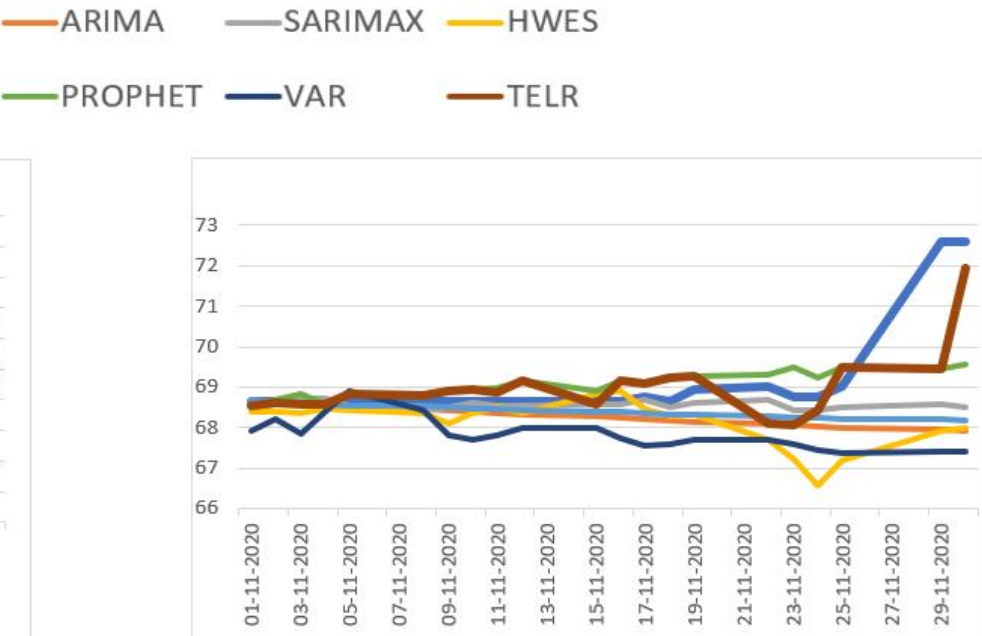

(b) 50-100 - Meghna Cement Company Limited

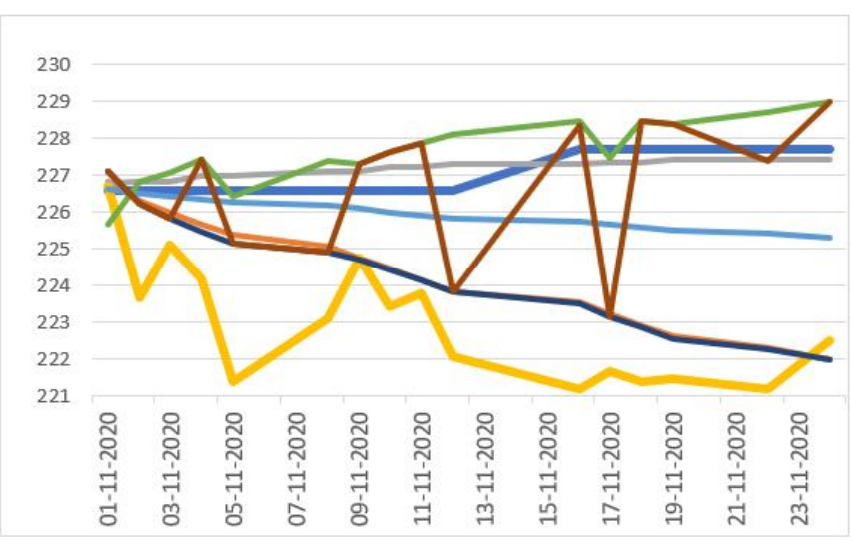

(d) 200-300 - Rahim Textile Company Limited

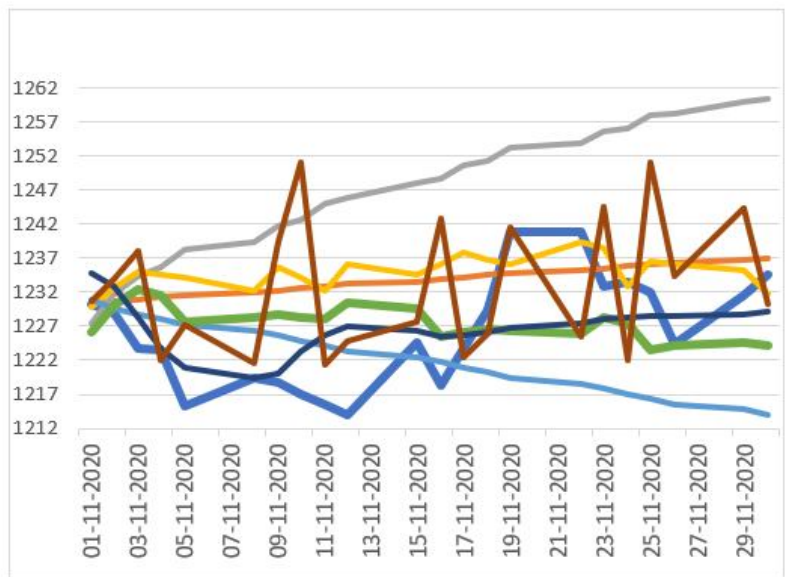

(f) Up to 400 - Linde Company Limited

Figure 2:Comparisons Graph of Actual Value vs Prediction for Various Time Series Model

\section{CONCLUSION \& FUTURE WORK}

A huge number of researches have shown that analysts with a variety of backgrounds are a major theme of forecasting. On this basis, we conclude that our proposed Meta model has much application value with a precise forecast.
The Meta model, presented in this paper combines a set of time series algorithms in stock price prediction as well as identifies which algorithm performs better for a particular company's stock data. Also, the noise value has been tuned based on the train data pattern considering the seasonal and growth nature. This yields the stock forecasting more 
accurately and lets the buyer/seller knows the pick time for selling/buying stock.

Though the Meta model considers the forecasting performance measurement works on comparing the actual and prediction value, hence, previous exponential or regression algorithm may work fine. But, the challenges of showing the trend like the upward or downwards nature or season of the stock price, our time series model (TELR) performs better than others.

The core decision point for an investor is to know the season of the highest and lowest peak of stock value for buying/selling stock. Hence, this stock price forecasting model will spread the advantage and eliminate the common lacking's of forecasting algorithm; finally, get an accurate forecasting result. Therefore, it will help investors to make wiser decisions without taking unnecessary risks.

In addition, these findings provide additional information about overcomes the challenge of prediction on unstable stock data as well as it can be regarded as one of the leading indicators of the stock market.

However, the parameters of the model (algorithm) could be dynamically changed while fitting data into it considering the error rate and seasonal effect of that algorithm. Also, a mathematical combination of different time series models' prediction for a particular date could come with a more accurate prophecy. In a word, considering other indicators of the stock market is needed to achieve good accuracy in the stock forecasting.

\section{ACKNOWLEDGEMENT}

This study is conducted and supported by Software Evolution and Re-Engineering Research (SERER) Lab.

\section{REFERENCES}

1. Agarwal, U., \&Sabitha, A. S. (2016, August). Time series forecasting of stock market index. In 2016 1st India International Conference on Information Processing (IICIP) (pp. 1-6). IEEE

2. Obert D. Edwards, John Magee, W.H.C. Bassetti, Technical Analysis of Stock Trends. CRC Press, Taylor \& Francis Group. 2007.

3. Idrees, S. M., Alam, M. A., \& Agarwal, P. (2019). A Prediction Approach for Stock Market Volatility Based on Time Series Data. IEEE Access, 7, 17287-17298.

4. Singh, Ritika, and Shashi Srivastava. "Stock Prediction Using Deep Learning." Multimedia Tools and Applications 76.18 (2017): 18569-18584.

5. Bollen, J., Mao, H., \& Zeng, X. (2011). Twitter mood predicts the stock market. Journal of computational science, 2(1), 1-8.

6. Wei, Liang-Ying. "A Hybrid ANFIS Model Based on Empirical Mode Decomposition for Stock Time Series Forecasting." Applied Soft Computing 42 (2016): 368-376.
7. Liu, H., \& Long, Z. (2020). An Improved Deep Learning Model for Predicting Stock Market Price Time Series. Digital Signal Processing, 102, 102741.

8. Fama, E. F. (1965). The Behavior Of Stock-Market Prices. The Journal of Business, 38, 34-105.

9. Taylor, Sean $\mathbf{J}$ and Letham, Benjamin. Forecasting at Scale. The American Statistician, Volume: 72, Issue: 1, pp 37-45, 2018.

10. Dimson, E. and Marsh, P. Volatility Forecasting Without Data-Snooping, Journal of Banking and Finance, 14,pp 399-421, 1990.

11. P. S. Kalekar, Time Series Forecasting Using Holt-Winters Exponential Smoothing, KanwalRekhi School of Information Technology, 2004.

12. Jane A. OuStephen H. Penman, Financial Statement Analysis And The Prediction of Stock Returns. Journal of Accounting and Economics, Volume 11, Issue 4, pp 295-329, November 1989.

13. Stock Bangladesh, Available: https://stockbangladesh.com, Last Accessed on 28th December, 2020

14. Fama, E. F. (1965). The Behavior Of Stock-Market Prices. The Journal of Business, 38, 34-105.

15. Dutta, A., Bandopadhyay, G., \& Sengupta, S. (2012). Prediction Of Stock Performance In The Indian Stock Market Using Logistic Regression. International Journal of Business and Information, 7(1), 105.

16. Devi, B. Uma, D. Sundar, and P. Alli. 2013. An Effective Time Series Analysis for Stock Trend Prediction Using Arima Model for Nifty Midcap-50. International Journal of Data Mining \& Knowledge Management Process.

17. Ariyo, A. A., Adewumi, A. O., \& Ayo, C. K. (2014, March). Stock Price Prediction Using TheARIMA Model. In 2014 UKSim-AMSS 16th International Conference on Computer Modelling and Simulation (pp. 106-112). IEEE.

18. Sharma, A., Bhuriya, D., \& Singh, U. (2017, April). Survey Of Stock Market Prediction Using Machine Learning Approach. In 2017 International conference of electronics, communication and aerospace technology (ICECA) (Vol. 2, pp. 506-509). IEEE.

19. Dey, S., Kumar, Y., Saha, S., \&Basak, S. (2016). Forecasting To Classification: Predicting The Direction Of Stock Market Price Using Xtreme Gradient Boosting. PESIT South Campus.

20. Tsantekidis, Avraam, et al. "Forecasting Stock Prices From The Limit Order Book Using Convolutional Neural Networks." 2017 IEEE 19th Conference on Business Informatics (CBI). Vol. 1. IEEE, 2017.

21. Samarawickrama, A. J. P., and T. G. I. Fernando. "A Recurrent Neural Network Approach In Predicting Daily Stock Prices An Application To The Sri Lankan Stock Market." 2017 IEEE International Conference on Industrial and Information Systems (ICIIS). IEEE, 2017. 
22. Selvin, Sreelekshmy, et al. "Stock Price Prediction Using LSTM, RNN AndCNN-Sliding Window Model." 2017 international conference on advances in computing, communications and informatics (icacci). IEEE, 2017.

23. Zhang, J., Cui, S., Xu, Y., Li, Q., \& Li, T. (2018). A Novel Data-Driven Stock Price Trend Prediction System. Expert Systems with Applications, 97, 60-69.

24. Totakura, V., Devasekhar, V., \& Sake, M. Prediction Of Stock Trend For Swing Trades Using Long Short-Term Memory Neural Network Model.

25. Kim, K. J. (2003). Financial Time Series Forecasting Using Support Vector Machines. Neurocomputing, 55(1-2), 307-319.

26. Kim, T., \& Kim, H. Y. (2019). Forecasting Stock Prices With A Feature Fusion LSTM-CNN Model Using Different Representations Of The Same Data. PloS one, 14(2), e0212320.

27. Yoshihara, A., Fujikawa, K., Seki, K. and Uehara, K., 2014, December. Predicting Stock Market Trends By Recurrent Deep Neural Networks. In Pacific rim international conference on artificial intelligence (pp. 759-769).

28. Nardo, M., Petracco-Giudici, M., \&Naltsidis, M. (2015). Walking Down Wall Street with A Tablet: A Survey of Stock Market Predictions Using the Web.

29. Cakra, Y. E., \&Trisedya, B. D. (2015, October). Stock Price Prediction Using Linear Regression Based On Sentiment Analysis. In 2015 international conference on advanced computer science and information systems (ICACSIS) (pp. 147-154). IEEE.

30. Pagolu, V. S., Reddy, K. N., Panda, G., \& Majhi, B. (2016, October). Sentiment Analysis of Twitter Data For Predicting Stock Market Movements. In 2016 international conference on signal processing, communication, power and embedded system (SCOPES) (pp. 1345-1350). IEEE.

31. Maqsood, H., Mehmood, I., Maqsood, M., Yasir, M., Afzal, S., Aadil, F., ... \& Muhammad, K. (2020). A Local And Global Event Sentiment Based Efficient Stock Exchange Forecasting Using Deep Learning. International Journal of Information Management, 50, 432-451.

32. Chen, T. L., \& Chen, F. Y. (2016). An Intelligent Pattern Recognition Model For Supporting Investment Decisions In Stock Market.Information Sciences, 346, 261-274.

33. Dai, Z., \& Zhu, H. (2020). Forecasting Stock Market Returns By Combining Sum-Of-The-Parts And Ensemble Empirical Mode Decomposition. Applied Economics, 52(21), 2309-2323.

34. Cervelló-Royo, R., Guijarro, F., \&Michniuk, K. (2015). Stock Market Trading Rule Based On Pattern Recognition And Technical Analysis: Forecasting the DJIA index with intraday data. Expert systems with Applications, 42(14), 5963-5975.
35. J, Kamalakannan and Sengupta, Indrani and Chaudhury, Snehaa, Stock Market Prediction Using Time Series Analysis, IADS International Conference on Computing, Communications \& Data Engineering (CCODE) 7-8 February 2018.

36. A. J. Conejo, M. A. Plazas, R. Espinola, and A. B. Molina, Day-AheadElectricity Price Forecasting Using The Wavelet Transform And ARIMA Models, IEEE Trans. Power Syst. Volume 20, Issue 2, pp 1035-1042, May 2005.

37. Vagropoulos, S. I., Chouliaras, G. I., Kardakos, E. G., Simoglou, C. K., \&Bakirtzis, A. G. (2016, April). Comparison OfSARIMAX, SARIMA, Modified SARIMA And ANN-Based Models For Short-Term PV Generation Forecasting. In 2016 IEEE International Energy Conference (ENERGYCON) (pp. 1-6). IEEE.

38. P. S. Kalekar, Time Series Forecasting Using Holt-Winters Exponential Smoothing, KanwalRekhi School of Information Technology, 2004.

39. Lewis, C. D. Industrial And Business Forecasting Methods: A Radical Guide To Exponential Smoothing And Curve Fitting. London; Boston: Butterworth Scientific, 1982.

40. Lütkepohl, H. (2006). Structural Vector Autoregressive Analysis For Cointegrated Variables. AllgemeinesStatistischesArchiv, 90(1), 75-88.

41. Md. Iftekharul Alam Efat, Rakibul Bashar, K. M. Imtiaz Uddin, TouhidBhuiyan. Trend Estimation OfStock Market: An Intelligent Decision System, International Conference on Cyber Security and Computer Science (ICONCS'18), pp 44-49, 18-20 October 2018.

42. R. K. Klimberg, G. P. Sillup, K. J. Boyle, and V. Tavva, "Forecasting Performance Measures-What Are Their Practical Meaning?" Advances in business and management forecasting, vol. 7, pp. 137-147,2010.

43. Tirea, M., \&Negru, V. (2013, June). Stock Market Analysis-Strongest Performing Stocks Influence On An Evolutionary Market. In Proceedings of the ITI 2013 35th International Conference on Information Technology Interfaces (pp. 263-270). IEEE.

44. Klimberg, R. K., \&Ratick, S. (2000, November). A New Measure Of Relative Forecast Error. In INFORMS Fall Meeting. 